\title{
Meta-analysis of Valve-in-Valve Transcatheter versus Redo Surgical Aortic Valve Replacement
}

\author{
Hisato Takagi ${ }^{1}$ Shohei Mitta ${ }^{1}$ Tomo Ando ${ }^{2}$ \\ ${ }^{1}$ Department of Cardiovascular Surgery, Shizuoka Medical Center, \\ Shizuoka, Japan \\ 2 Department of Cardiology, Detroit Medical Center, Detroit, United States \\ Address for correspondence Hisato Takagi, MD, PhD, Department of \\ Cardiovascular Surgery, Shizuoka Medical Center, 762-1 Nagasawa, \\ Shimizu-cho, Sunto-gun, Shizuoka 411-8611, Japan \\ (e-mail: kfgth973@ybb.ne.jp). \\ Thorac Cardiovasc Surg 2019;67:243-250.
}

\begin{abstract}
Keywords

- degenerated aortic valve bioprostheses

- meta-analysis

- redo surgical aortic valve replacement

- valve-in-valve transcatheter aortic valve implantation

Objective The objective of this study was to determine whether valve-in-valve transcatheter aortic valve implantation (VIV-TAVI) is associated with better survival than redo surgical aortic valve replacement (SAVR) in patients with degenerated aortic valve bioprostheses, and we performed a meta-analysis of comparative studies.

Methods To identify all comparative studies of VIV-TAVI versus redo SAVR; MEDLINE, Embase, and the Cochrane Central Register of Controlled Trials were searched through October 2017. For each study, data regarding all-cause mortality in both the VIV-TAVI and redo SAVR groups were used to generate odds ratios (ORs). To assess selection bias, we generated ORs and (standardized) mean differences (MDs) for baseline characteristics. Study-specific estimates were combined in the random-effects model. Results Of 446 potentially relevant articles screened initially, 6 reports of retrospective comparative studies enrolling a total of 498 patients were identified. Pooled analyses of baseline characteristics demonstrated no statistically significant differences in the proportion of women, patients with diabetes mellitus, patients with coronary artery disease, and patients with baseline New York Heart Association functional class of $\geq \mathrm{III}$; baseline ejection fraction; and predicted mortality between the VIV-TAVI and redo SAVR groups. Patients in the VIV-TAVI group, however, were significantly older (MD, 4.20 years) and had undergone prior coronary artery bypass grafting more frequently $(O R, 2.19)$ than those in the redo SAVR group. Main pooled analyses demonstrated no statistically significant differences in early (30 days or inhospital) $(\mathrm{OR}, 0.91 ; p=0.83)$ and midterm (180 days -3 years) all-cause mortalities (OR, 1.42; $p=0.21$ ) between the VIV-TAVI and redo SAVR groups.

Conclusion In patients with degenerated aortic valve bioprostheses, especially elderly or high-risk patients, VIV-TAVI could be a safe, feasible alternative to redo SAVR. The lack of randomized data and differences in baseline characteristics in the present analysis emphasize the need for prospective randomized trials.
\end{abstract}

\section{Introduction}

The use of valve-in-valve (VIV) transcatheter aortic valve implantation (TAVI) (VIV-TAVI) for the treatment of high-risk patients with degenerated aortic bioprostheses is associated with relatively low rates of mortality and major complica-

received

February 15, 2018

accepted after revision

July 2, 2018

published online

August 16, 2018

tions, improved hemodynamics and excellent improvement in functional and quality of life outcomes at 1 year, ${ }^{1}$ whereas redo surgical aortic valve replacement (SAVR) is now performed with acceptable operative mortality of $4.6 \%{ }^{2}$ Although mortality and morbidity are high compared with primary SAVR, the rates of stroke, vascular complications,

(c) 2019 Georg Thieme Verlag KG Stuttgart · New York
DOI https://doi.org/ 10.1055/s-0038-1668135. ISSN 0171-6425. 
and postoperative aortic regurgitation are low. ${ }^{2}$ To our knowledge, however, there have been a few comparative studies of VIV-TAVI and redo SAVR to date. Because of its less invasiveness than redo SAVR, VIV-TAVI is expected to reduce mortality in patients with degenerated aortic valve bioprostheses who are at high surgical risk in the extreme. To determine whether VIV-TAVI is associated with better survival than redo SAVR, we herein performed a meta-analysis of comparative studies of VIV-TAVI versus redo SAVR.

\section{Materials and Methods}

All comparative studies of VIV-TAVI versus redo SAVR enrolling patients with degenerated aortic valve bioprostheses were identified using a two-level search strategy. First, databases including MEDLINE, Embase, and the Cochrane Central Register of Controlled Trials were searched through October 2017 using Web-based search engines (PubMed and Ovid). Second, relevant studies were identified through a manual search of secondary sources including references of initially identified articles and a search of reviews and commentaries. All references were downloaded for consolidation, elimination of duplicates, and further analysis. Search terms included valve-in, TAVI-in, or TAV-in; redo, re-do, reoperation, re-operation, reoperative, re-operative, re-surgery, or re-surgical; and aortic valve.

Studies considered for inclusion met the following criteria: the design was a comparative study; the study population was patients with degenerated aortic valve bioprostheses; patients were assigned to VIV-TAVI versus redo SAVR; and main outcomes included all-cause mortality. Data regarding detailed inclusion criteria, device type, duration of follow-up, and allcause mortality were abstracted (as available) from each individual study. We focused on mortality as an outcome of interest in the present meta-analysis. Data were extracted in duplicate by two investigators (H.T. and S.M.) and independently verified by a third investigator (T.A.). Disagreements were resolved by consensus.

We conducted a meta-analysis of summary statistics from the individual studies because detailed patient-level data were not available for all studies. For each study, data regarding allcause mortality in both the VIV-TAVI and redo SAVR groups were used to generate odds ratios (ORs) and 95\% confidence intervals (CIs). To assess selection bias (differences in baseline characteristics of individuals in different intervention groups), we generated ORs for proportions (\%) of women, patients with diabetes mellitus (DM), patients with coronary artery disease (CAD), patients undergoing prior coronary artery bypass grafting (CABG), and patients with baseline New York Heart Association (NYHA) functional class of $\geq \mathrm{III}$; mean differences (MDs) for ages (year) and baseline left ventricular ejection fraction (LVEF [\%]); and a standardized MD (SMD) for predicted mortality (\%).

Study-specific estimates were combined using inverse variance-weighted averages of logarithmic ORs and MDs or SMDs in the random-effects model. Publication bias was assessed graphically using a funnel plot and mathematically using an adjusted rank-correlation test of Begg and Mazumdar ${ }^{3}$ and a linear regression test of Egger et al. ${ }^{4}$ All analyses were conducted using Review Manager version 5.3 (available at: http:// community.cochrane.org/tools/review-production-tools/revman-5) and Prometa 3 (available at: https://idostatistics.com/ prometa3/).

\section{Results}

As outlined in -Supplementary Fig. S1 (available online only), of 446 potentially relevant articles screened initially, 6 reports $^{5-10}$ of comparative studies of VIV-TAVI versus redo SAVR enrolling a total of 498 (VIV-TAVI, 254; redo SAVR, 244) patients with degenerated aortic valve bioprostheses were identified and included ( - Table 1 ). All were retrospective observational studies including two matched studies. ${ }^{5,10}$ Patients were matched 1:1 on Society of Thoracic Surgeons Predicted Risk Of Mortality (STS-PROM) scores followed by sex, age, and year of procedure in a study, ${ }^{5}$ and propensity scores among patients undergoing either VIV-TAVI or redo SAVR were matched to obtain matched pairs of patients in another study. ${ }^{10}$ In the other four studies, ${ }^{6-9}$ no adjustment was performed. Types of degenerated bioprostheses are summarized in - Supplementary Table S1 (available online only). No concomitant procedure was performed in the redo SAVR group of four studies. ${ }^{5,6,8,9}$ In a study, ${ }^{7}$ concomitant CABG, mitral valve surgery, and others were performed in 20,40 , and $8.0 \%$ of patients, respectively. In another study, ${ }^{10}$ concomitant CABG was performed in $26.9 \%$ of patients. Some end points except for mortality are summarized in -Table 2.

Pooled analyses of baseline characteristics (- Table $\mathbf{3}$ ) demonstrated no statistically significant differences in the proportion of women (44.9 vs. 39.3\%; $p=0.37$; -Supplementary Fig. S2, available online only), patients with DM (21.1 vs. $15.1 \% ; p=0.05$; -Supplementary Fig. S3, available online only), patients with CAD (43.8 vs. 23.9\%; $p=0.12$; - Supplementary Fig. S4, available online only), and patients with baseline NYHA functional class of $\geq$ III ( 86.7 vs. $79.5 \% ; p=0.22$; - Supplementary Fig. S5, available online only); baseline LVEF (MD, -2.07\%; $p=0.39 ; \quad$-Supplementary Fig. S6, available online only); and predicted mortality (SMD, $-0.03 ; p=0.91$; -Supplementary Fig. S7, available online only) between the VIV-TAVI and redo SAVR groups. Patients in the VIVTAVI group, however, were significantly older than those in the redo SAVR group (MD, 4.20 years; $p=0.004 ;$ - Supplementary Fig. S8, available online only). Furthermore, significantly more patients undergone prior CABG in the VIV-TAVI group than in the redo SAVR group (36.7 vs. 21.8\%; OR, 2.19; $p=0.005$; - Supplementary Fig. S9, available online only).

Main pooled analyses demonstrated no statistically significant differences in early (30 days or in-hospital) (OR, $0.91 ; p=0.83$ ) and midterm (180 days-3 years) all-cause mortalities (OR, $1.42 ; p=0.21$ ) between the VIV-TAVI and redo SAVR groups (- Table 4 ; - Fig. $\mathbf{1}$ ).

To assess publication bias in the main meta-analyses, we generated a funnel plot of the OR versus the standard error 


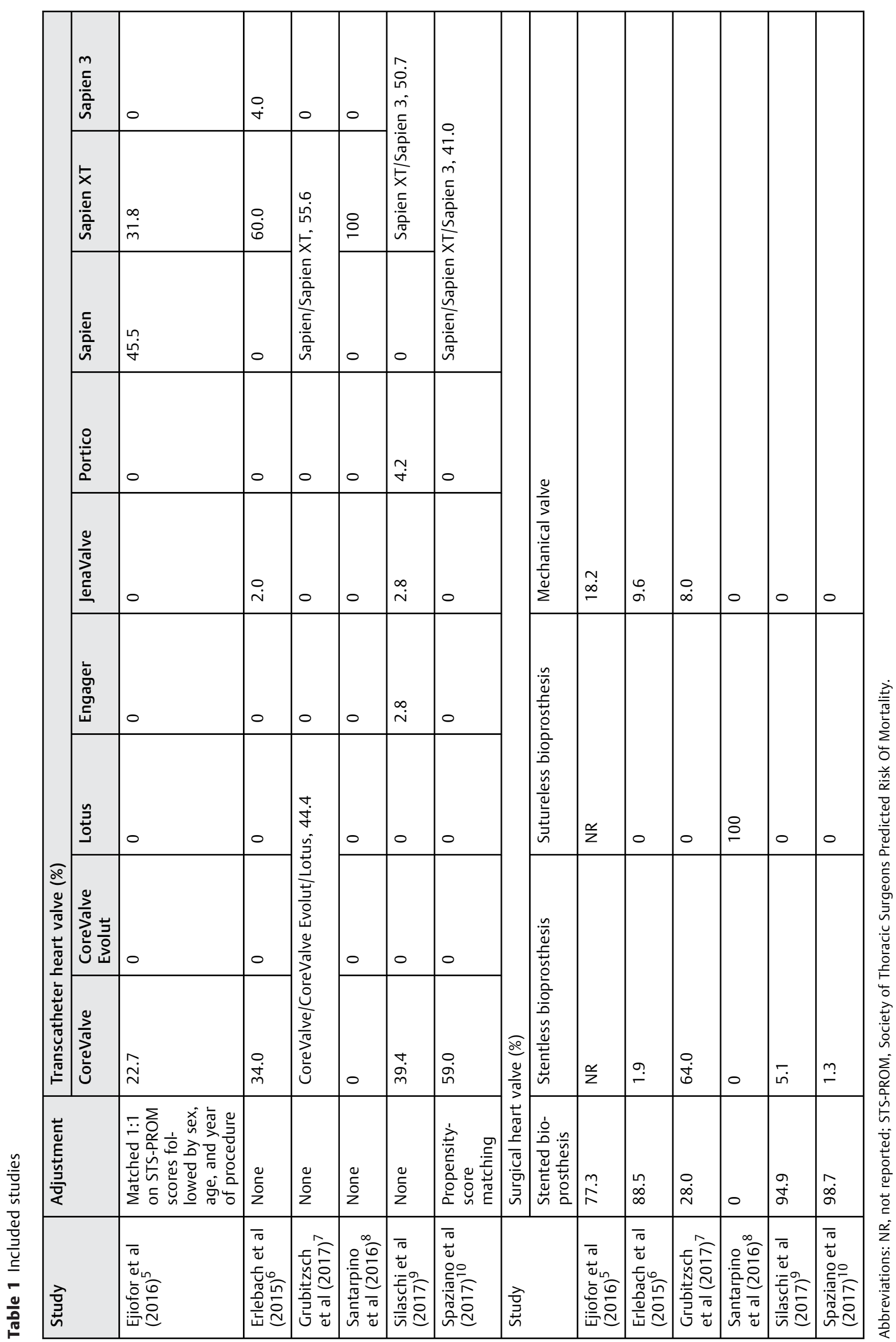




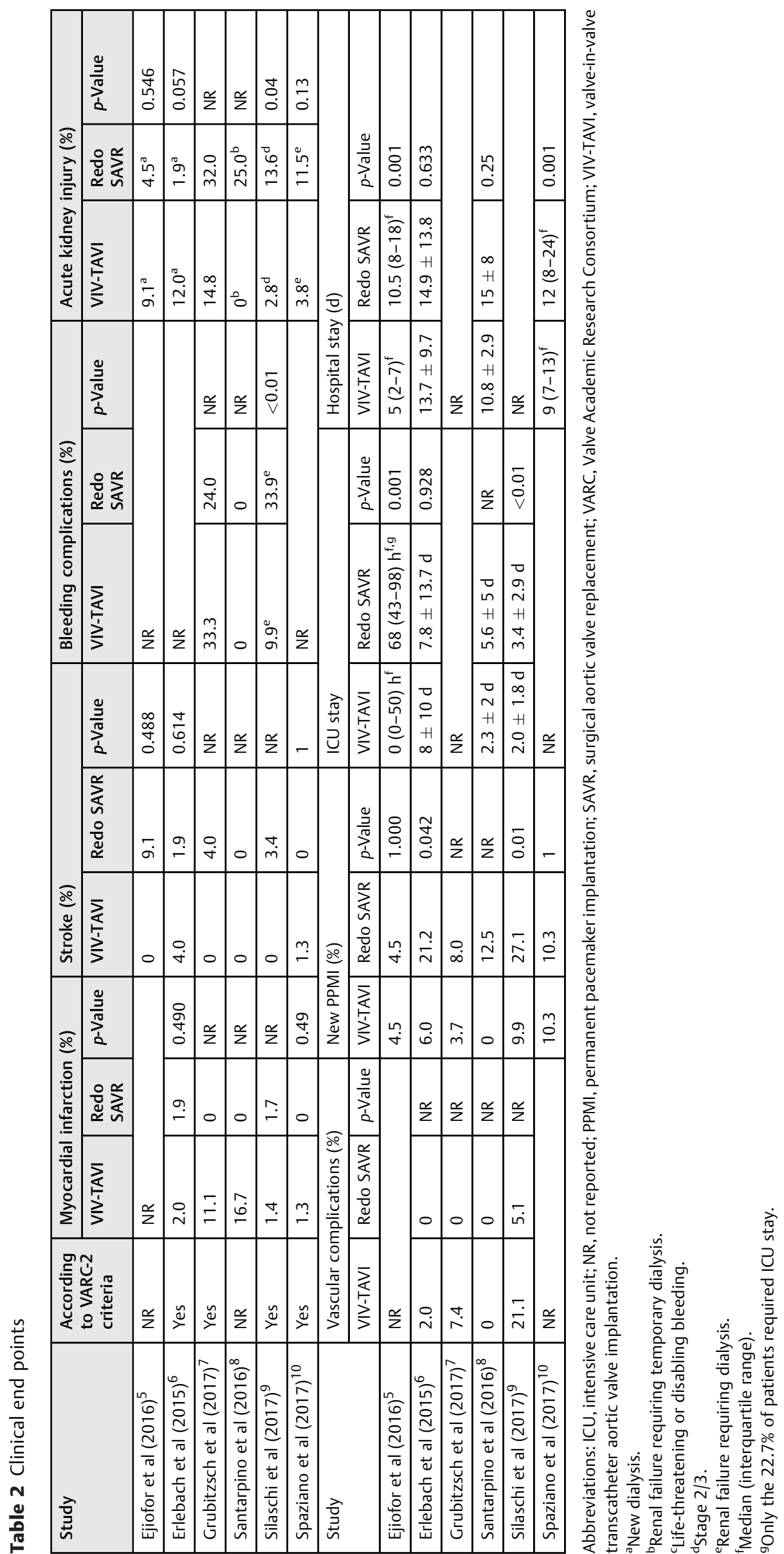




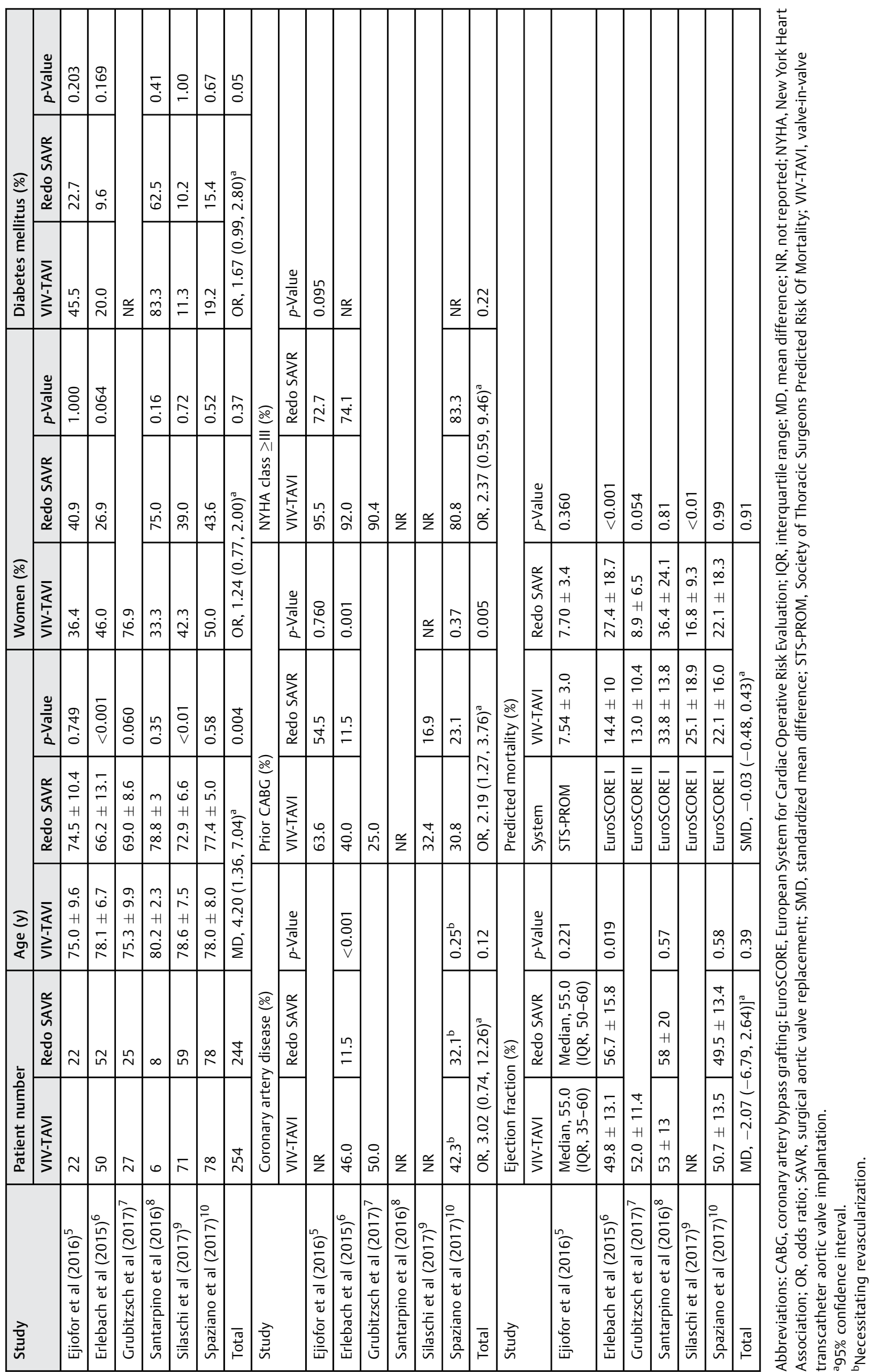


Table 4 Early and midterm all-cause mortalities

\begin{tabular}{|c|c|c|c|c|c|c|}
\hline \multirow[t]{2}{*}{ Study } & \multicolumn{3}{|c|}{ Early all-cause mortality (\%) } & \multicolumn{3}{|c|}{ Midterm all-cause mortality (\%) } \\
\hline & Follow-up & VIV-TAVI & Redo SAVR & Follow-up & VIV-TAVI & Redo SAVR \\
\hline Ejiofor et al $(2016)^{5}$ & Operative & 0 & 4.5 & $3 y$ & $21.3^{\mathrm{a}}$ & $23.7^{\mathrm{a}}$ \\
\hline Erlebach et al $(2015)^{6}$ & $30 \mathrm{~d}$ & 4.0 & 0 & $1 \mathrm{y}$ & $4^{\mathrm{a}}$ & $17^{\mathrm{a}}$ \\
\hline Grubitzsch et al $(2017)^{7}$ & $30 \mathrm{~d}$ & 11.1 & 8.0 & $1 \mathrm{y}$ & 18.5 & 16.0 \\
\hline Santarpino et al $(2016)^{8}$ & In-hospital & 0 & 0 & $21 \pm 13 \mathrm{mo}$ & 0 & 0 \\
\hline Silaschi et al $(2017)^{9}$ & $30 \mathrm{~d}$ & 4.2 & 5.1 & $180 \mathrm{~d}$ & 10.9 & 7.8 \\
\hline Spaziano et al $(2017)^{10}$ & $30 \mathrm{~d}$ & 3.8 & 6.4 & $1 \mathrm{y}$ & 11.5 & 12.8 \\
\hline Total & \multicolumn{3}{|c|}{ OR, $0.91(0.39,2.13)^{\mathrm{b}} ; p=0.83$} & \multicolumn{3}{|c|}{ OR, $1.42(0.82,2.46)^{\mathrm{b}} ; p=0.21$} \\
\hline
\end{tabular}

Abbreviations: OR, odds ratio; SAVR, surgical aortic valve replacement; VIV-TAVI, valve-in-valve transcatheter aortic valve implantation. aKaplan-Meier's estimate.

${ }^{\mathrm{b}} 95 \%$ confidence interval.

for each study (- Supplementary Figs. S10 and S11, available online only). There was no evidence of significant publication bias: $p$ for early all-cause mortality $=0.348$ and 0.494 (-Supplementary Fig. S10, available online only) and $p$ for midterm all-cause mortality $=0.573$ and 0.493 (-Supplementary Fig. S11, available online only) by the tests of Begg and Mazumdar ${ }^{3}$ and Egger et $a l,{ }^{4}$ respectively.

\section{Discussion}

The results of the present meta-analysis suggest no difference in survival between VIV-TAVI and redo SAVR in patients with degenerated aortic valve bioprostheses. Although the proportion of women, patients with DM, patients with base- line NYHA functional class of $\geq$ III, baseline LVEF, and predicted mortality were similar between the VIV-TAVI and redo SAVR groups, patients in the VIV-TAVI group were older than those in the redo SAVR group.

Adding that older patients underwent VIV-TAVI, the following findings could explain similar survival between VIVTAVI and redo SAVR despite less invasiveness of VIV-TAVI than redo SAVR. In the study by Ejiofor et al, ${ }^{5} 22.7 \%$ of VIVTAVI patients had mild paravalvular leaks compared with none in the SAVR group $(p=0.048)$. Erlebach et $\mathrm{al}^{6}$ showed that the rate of postprocedural new dialysis (12.0 vs. $1.9 \%$; $p=0.057)$, paravalvular leak (18.0 vs. $0 \%$ ), and mean aortic valve gradient $(18.8 \pm 8.7$ vs. $13.8 \pm 5.4 \mathrm{~mm} \mathrm{Hg} ; p=0.008)$ were higher in the VIV-TAVI group compared with the redo
Odds Ratio

Study or Subgroup Weight IV, Random, $95 \% \mathrm{CI}$

\begin{tabular}{lrr}
\hline 1.10 .1 Early all-cause mortality & \\
Ejiofor 2016 & $6.8 \%$ & $0.32[0.01,8.25]$ \\
Erlebach 2015 & $7.7 \%$ & $5.41[0.25,115.58]$ \\
Grubitzsch 2017 & $20.5 \%$ & $1.44[0.22,9.41]$ \\
Santarpino 2016 & $4.4 \%$ & $1.31[0.02,75.13]$ \\
Silaschi 2016 & $26.9 \%$ & $0.82[0.16,4.24]$ \\
Spaziano 2017 & $33.6 \%$ & $0.58[0.13,2.53]$ \\
Subtotal (95\% Cl) & $100.0 \%$ & $\mathbf{0 . 9 1}[0.39,2.13]$ \\
Heterogeneity. Tau ${ }^{2}=0.00 ; \mathrm{Chi}^{2}=2.33, \mathrm{df}=5(\mathrm{P}=0.80) ; \mathrm{l}^{2}=0 \%$ \\
Test for overall effect: $\mathrm{Z}=0.22(\mathrm{P}=0.83)$
\end{tabular}

1.10.2 Midterm all-cause mortality

Ejiofor 2016

Erlebach 2015

Grubitzsch 2017

Santarpino 2016

Silaschi 2016

Spaziano 2017

Subtotal $(95 \% \mathrm{CI})$

$23.4 \%$

$12.1 \%$

$1.61[0.52,4.99]$

$14.4 \% \quad 1.19[0.28,5.06]$

$1.8 \% \quad 1.31[0.02,75.13]$

$15.8 \% 1.43[0.36,5.69]$

$32.5 \% \quad 0.89[0.34,2.32]$

Heterogeneity. $\mathrm{Tau}^{2}=0.00 ; C \mathrm{Ci}^{2}=3.43, \mathrm{df}=5(\mathrm{P}=0.63) ; \mathrm{l}^{2}=0 \%$

Test for overall effect: $z=1.26(P=0.21)$
Odds Ratio

IV, Random, 95\% CI

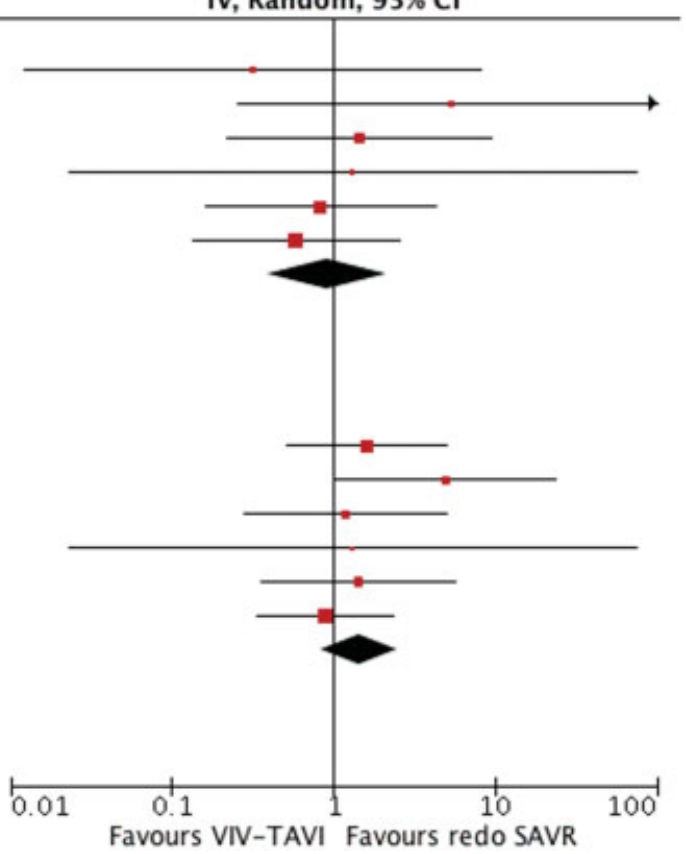

Fig. 1 Early and midterm all-cause mortalities. CI, confidence interval; IV, inverse variance; SAVR, surgical aortic valve replacement; VIV-TAVI, valve-in-valve transcatheter aortic valve implantation. 
SAVR group. At follow-up ( $21 \pm 13$ months) echocardiographic evaluation in the study by Santarpino et $\mathrm{al}^{8}{ }^{8} 87.5 \%$ of patients had no patient-prosthesis mismatch (PPM) and only $12.5 \%$ had mild/moderate PPM in the redo SAVR group, whereas only $16.7 \%$ had no PPM, 50.0\% had mild/moderate PPM, and 33.3\% had severe PPM in the VIV-TAVI group. The mean indexed effective orifice area (EOA) was $0.96 \pm 0.08$ versus $0.71 \pm 0.15 \mathrm{~cm}^{2} / \mathrm{m}^{2}$ in the redo SAVR versus VIV-TAVI group, respectively $(p=0.001) .{ }^{8}$ Silaschi et al ${ }^{9}$ showed that a mean gradient of $\geq 20 \mathrm{~mm} \mathrm{Hg}$ was present in $46.5 \%$ after VIVTAVI versus $5.1 \%$ after SAVR $(p<0.01)$ and the proportion of severe PPM (defined by indexed EOA $\leq 0.65 \mathrm{~cm}^{2} / \mathrm{m}^{2}$ ) tended to be higher compared with that of redo SAVR (14.1 vs. 3.4\%; $p=0.06)$. Decrease in transprosthetic gradients was stronger $(p=0.01)$ after redo SAVR $(25.0 \mathrm{~mm} \mathrm{Hg})$ than after VIVTAVI $(13.3 \mathrm{~mm} \mathrm{Hg}){ }^{9}$ At 30 days in the study by Spaziano et al, ${ }^{10}$ redo SAVR was associated with a lower mean gradient compared with VIV-TAVI $(14.3 \pm 6.2$ vs. $18.1 \pm 7.4 \mathrm{~mm} \mathrm{Hg}$; coefficient, $3.78 \mathrm{~mm} \mathrm{Hg}$; $95 \% \mathrm{Cl}, \quad 0.95-6.60 \mathrm{~mm} \mathrm{Hg}$; $p=0.01$ ) and moderately elevated postprocedural mean gradients $>20 \mathrm{~mm} \mathrm{Hg}$ were more frequently reported after VIV-TAVI than after redo SAVR (36 vs. $17 \%$; OR, 3.74; $95 \% \mathrm{CI}$, $1.48-9.41 ; p=0.04)$. The above-mentioned findings unfavorable for VIV-TAVI may offset its less invasiveness than redo SAVR and affect early and midterm survival.

Although baseline CAD may affect follow-up mortality after VIV-TAVI and/or redo SAVR, there was no difference in the proportion of patients with CAD between the VIV-TAVI and redo SAVR groups ( Supplementary Fig. S4, available online only). More patients, however, had undergone prior CABG in the VIV-TAVI group than in the redo SAVR group (-Supplementary Fig. 59, available online only). Only Grubitzsch et $\mathrm{al}^{7}$ (in the six studies included in the present metaanalysis) reported data on a patent internal mammary artery (IMA) graft, and VIV-TAVI patients presented more frequently with a patent IMA graft than redo SAVR (33.3 vs. 8.0\%; $p=0.040$ ). Patients undergoing SAVR after CABG in the presence of a patent bypass graft (especially left IMA to left anterior descending artery graft) are at high risk of graft injury; therefore, surgeons should specifically consider to prevent graft injury. ${ }^{11}$ Injury of a patent left IMA graft during dissection can have serious consequences, and the rate of injury has been reported to be 5 to $40 \%$, with perioperative mortality to be 9 to $50 \% .{ }^{12-14}$ Therefore, surgeons may avoid redo SAVR in patients with prior CABG, in whom VIV-TAVI can be selected.

In the PARTNER 2 Valve-in-Valve Registry, ${ }^{1}$ a multivariate Cox's proportional hazard regression model was used to assess the adjusted association between mortality and risk factors: STS-PROM, labeled valve size, transcatheter heart valve (THV) size, mean gradient $\geq 20 \mathrm{~mm} \mathrm{Hg}$, and severe PPM. Although there were no significant differences in mortality comparing previous surgical valve sizes $(15.5 \%$ for $21 \mathrm{~mm}$ vs $11.4 \%$ for $>21 \mathrm{~mm}$; hazard ratio [HR], 1.39; $95 \% \mathrm{CI}, 0.73-2.63 ; p=0.3156)$, mortality was significantly greater at 1 year with a larger postprocedural mean gradient ( $16.7 \%$ for $\geq 20 \mathrm{~mm} \mathrm{Hg}$ vs. $7.7 \%$ for $<20 \mathrm{~mm} \mathrm{Hg}$; HR, 2.27 ; $95 \%$ CI, 1.16-4.46; $p=0.0140) .{ }^{1}$ Trends toward increased mortality in patients with PPM (10.3\% for severe PPM vs. $9.7 \%$ for moderate PPM vs. $6.9 \%$ for no PPM) were not significant $(p=0.8617) .{ }^{1}$ Also, in the studies ${ }^{6,9}$ included in the present meta-analysis, postprocedural gradients were higher after VIV-TAVI than redo SAVR $(18.8 \pm 8.7$ vs. $13.8 \pm 5.4 \mathrm{~mm} \mathrm{Hg}$ $[p=0.008],{ }^{6} 19.7$ vs. $\left.12.2 \mathrm{~mm} \mathrm{Hg}[p=0.01]^{9}\right)$. Careful patient selection, preoperative image assessment, and accurate valve deployment during the procedure may be required to reduce postprocedure gradients after VIV-TAVI. ${ }^{5}$ Redo SAVR rather than VIV-TAVI may be preferred in younger patients with degenerated aortic valve bioprostheses. Indeed, patients undergoing redo SAVR were younger than those undergoing VIV-TAVI in three $e^{6,7,9}$ of the six studies included in the present study ( - Table 2 ), which was confirmed in our meta-analytic evaluation (-Supplementary Fig. 58, available online only).

In the Global Valve-in-Valve Registry ${ }^{15}$ including 202 patients with degenerated bioprostheses, there were no significant differences between the CoreValve and Edwards SAPIEN groups in mortality, major vascular complication, or stroke at 30 days and 1 year survival. Implantation of Edwards SAPIEN versus CoreValve, however, was an independent predictor for high postprocedural gradients $(p=0.02)$. The difference is probably secondary to the fundamental dissimilarity between the devices (intra-annular and supra-annular functioning part of Edwards SAPIEN and CoreValve, respectively), which suggests that CoreValve depends much less on surgical bioprosthesis dimensions and its functioning part may have larger potential orifice area. ${ }^{15}$ High implantation inside failed bioprostheses is also a strong independent correlate of lower postprocedural gradients in both self-expandable and balloon-expandable THVs. ${ }^{16}$ In the Valve-in-Valve International Data registry ${ }^{16}$ evaluating 292 consecutive patients, the strongest independent correlate for lower gradients after VIV-TAVI was high device position $(p=0.001)$ in addition to CoreValve Evolut (vs. SAPIEN XT) use $(p=0.02)$, which suggests that it would be possible to obtain better hemodynamic results in VIV-TAVI if the THV functions above the surgical valve stent. ${ }^{17}$

Patients undergoing VIV-TAVI are particularly at risk for PPM because the TAVI prosthesis is implanted within the frame of the previous surgical bioprosthetic valve, thereby reducing the maximum EOA achieved with the new valve. ${ }^{18}$ There have recently been a few publications on the concept of fracturing the surgical bioprosthetic valve ring with a highpressure balloon inflation to dilate the surgical valve and permit further expansion of the THV. ${ }^{18-20}$ For the combined cohort, the mean gradient was reduced from $41 \mathrm{~mm} \mathrm{Hg}$ preprocedure to $11 \mathrm{~mm} \mathrm{Hg}$ after bioprosthetic valve fracture and VIV-TAVI, which corresponds to an improvement in EOA from 0.75 to $1.7 \mathrm{~cm}^{2}{ }^{2}{ }^{18-20}$ The benefit of bioprosthetic valve fracture to improve the procedural results of VIV-TAVI is evident, whereas these patients would have been left with a suboptimal EOA and gradient with VIV-TAVI alone. ${ }^{18}$

\section{Limitations}

Our analysis must be viewed in the context of its limitations. First, we used only data from retrospective observational 
comparative studies, not randomized controlled trials. Although the proportion of women, patients with DM, patients with baseline NYHA functional class of $\geq$ III, baseline LVEF, and predicted mortality were similar between the VIVTAVI and redo SAVR groups, patients in the VIV-TAVI group were older than those in the redo SAVR group. In studies included in the present meta-analysis, patients were preselected by a heart team, and therefore, the groups are biased. Second, our results may be influenced by publication bias. This risk was minimized through an exhaustive search of the available literature. Although the statistical tests did not indicate publication bias, there is clearly limited power to detect such bias, given the small number of studies examined.

\section{Conclusion}

In patients with degenerated aortic valve bioprostheses, especially elderly or high-risk patients, VIV-TAVI could be a safe and feasible alternative to redo SAVR. The lack of randomized data and differences in baseline characteristics in the present analysis emphasize the need for prospective randomized trials.

Conflict of Interest

None declared.

\section{References}

1 Webb JG, Mack MJ, White JM, et al. Transcatheter aortic valve implantation within degenerated aortic surgical bioprostheses: PARTNER 2 Valve-in-Valve Registry. J Am Coll Cardiol 2017;69 (18):2253-2262

2 Kaneko T, Vassileva CM, Englum B, et al. Contemporary outcomes of repeat aortic valve replacement: a benchmark for transcatheter valve-in-valve procedures. Ann Thorac Surg 2015;100(04): 1298-1304, discussion 1304

3 Begg CB, Mazumdar M. Operating characteristics of a rank correlation test for publication bias. Biometrics 1994;50(04):1088-1101

4 Egger M, Davey Smith G, Schneider M, Minder C. Bias in metaanalysis detected by a simple, graphical test. BMJ 1997;315 (7109):629-634

5 Ejiofor JI, Yammine M, Harloff MT, et al. Reoperative surgical aortic valve replacement versus transcatheter valve-in-valve replacement for degenerated bioprosthetic aortic valves. Ann Thorac Surg 2016;102(05):1452-1458

6 Erlebach M, Wottke M, Deutsch MA, et al. Redo aortic valve surgery versus transcatheter valve-in-valve implantation for fail- ing surgical bioprosthetic valves: consecutive patients in a singlecenter setting. J Thorac Dis 2015;7(09):1494-1500

7 Grubitzsch H, Zobel S, Christ T, et al. Redo procedures for degenerated stentless aortic xenografts and the role of valvein-valve transcatheter techniques. Eur J Cardiothorac Surg 2017; 51(04):653-659

8 Santarpino G, Pietsch LE, Jessl J, et al. Transcatheter aortic valvein-valve implantation and sutureless aortic valve replacement: two strategies for one goal in redo patients. Minerva Cardioangiol 2016;64(06):581-585

9 Silaschi M, Wendler O, Seiffert M, et al. Transcatheter valve-invalve implantation versus redo surgical aortic valve replacement in patients with failed aortic bioprostheses. Interact Cardiovasc Thorac Surg 2017;24(01):63-70

10 Spaziano M, Mylotte D, Thériault-Lauzier P, et al. Transcatheter aortic valve implantation versus redo surgery for failing surgical aortic bioprostheses: a multicentre propensity score analysis. EuroIntervention 2017;13(10):1149-1156

11 Kaneko T, Nauta F, Borstlap W, McGurk S, Rawn JD, Cohn LH. The "no-dissection" technique is safe for reoperative aortic valve replacement with a patent left internal thoracic artery graft. J Thorac Cardiovasc Surg 2012;144(05):1036-1040

12 Elami A, Laks H, Merin G. Technique for reoperative median sternotomy in the presence of a patent left internal mammary artery graft. J Card Surg 1994;9(02):123-127

13 Coltharp WH, Decker MD, Lea JW IV, et al. Internal mammary artery graft at reoperation: risks, benefits, and methods of preservation. Ann Thorac Surg 1991;52(02):225-228, discussion 229

14 Ivert TS, Ekeström S, Péterffy A, Welti R. Coronary artery reoperations. Early and late results in 101 patients. Scand J Thorac Cardiovasc Surg 1988;22(02):111-118

15 Dvir D, Webb J, Brecker S, et al. Transcatheter aortic valve replacement for degenerative bioprosthetic surgical valves: results from the Global Valve-in-Valve Registry. Circulation 2012;126(19):2335-2344

16 Simonato M, Webb J, Kornowski R, et al. Transcatheter replacement of failed bioprosthetic valves: large multicenter assessment of the effect of implantation depth on hemodynamics after aortic valve-in-valve. Circ Cardiovasc Interv 2016;9(06):e003651

17 Azadani AN, Jaussaud N, Matthews PB, et al. Valve-in-valve implantation using a novel supravalvular transcatheter aortic valve: proof of concept. Ann Thorac Surg 2009;88(06):1864-1869

18 Saxon JT, Allen KB, Cohen DJ, Chhatriwalla AK. Bioprosthetic valve fracture during valve-in-valve TAVR: bench to bedside. Interv Cardiol 2018;13(01):20-26

19 Chhatriwalla AK, Allen KB, Saxon JT, et al. Bioprosthetic valve fracture improves the hemodynamic results of valve-in-valve transcatheter aortic valve replacement. Circ Cardiovasc Interv 2017;10(07):e005216

20 Nielsen-Kudsk JE, Andersen A, Therkelsen CJ, et al. High-pressure balloon fracturing of small dysfunctional Mitroflow bioprostheses facilitates transcatheter aortic valve-in-valve implantation. EuroIntervention 2017;13(09):e1020-e1025 